\title{
Structural properties of (B3) TIP under pressure
}

\author{
Salah Daoud $^{1}$, Nadhira Bioud ${ }^{2}$ \\ ${ }^{1}$ Faculté des Sciences et de la Technologie, Université de Bordj Bou Arreridj, 34000, Algeria \\ ${ }^{2}$ Laboratoire d'Optoélectronique et Composants, Université Ferhat Abbes- Sétif, 19000, Algeria \\ *Corresponding author E-mail: salah_daoud07@yahoo.fr
}

Copyright () 2014 Salah Daoud, Nadhira Bioud. This is an open access article distributed under the Creative Commons Attribution License, which permits unrestricted use, distribution, and reproduction in any medium, provided the original work is properly cited.

\begin{abstract}
We present an Ab-initio study of the pressure effect on the structural properties of thallium phosphide (TlP) compound. The plane-wave pseudopotential approach to the density functional theory (DFT) within the local density approximation (LDA) implemented in Abinit code was used. The equilibrium lattice parameter, bulk modulus and its pressure derivatives, crystal density, near-neighbor and nearest-neighbor distances are calculated. The threshold pressure of the phase transition, the volume collapses and the Debye temperature are also obtained. Our results are in general in very good agreement with the previous results of the literature.
\end{abstract}

Keywords: PP-PW Approach, Structural Properties, TlP Compound.

\section{Introduction}

Semiconductor materials (silicon, germanium, selenium; lead telluride; lead sulfide, silicon carbide...etc.) are usually used to fabricate and elaborate the majority of electronic devices: modulators, detectors, photocells, transistors and diodes ...etc. In view of their important properties for eventual technological applications, $\mathrm{A}^{\mathrm{III}} \mathrm{B}^{\mathrm{V}}$ group compounds have attracted increasing research interest in the last few years. Much attention has been also given to the study of the different physical properties of binary tetrahedral semiconductors because of their potential applications in several areas, such as linear and nonlinear optics, solar cells, light-emitting diodes, etc... [1]. It has been suggested that the optoelectronic devices based on thallium-V compounds have advantages over the II-VI semiconductor devices, for many interesting applications, and especially in optical communication systems in the near-infrared wavelength region [2]. It is therefore important to understand the intrinsic physical properties of the thallium-V compounds, and to know how to select the appropriate materials for desired technological applications.

The structural stability of the thallium-V compounds: TIX (X=N, P, As, Sb, and Bi) was studied by Saidi-Houat et al. [3]. They found that the cubic zincblende (B3) structure of thallium phosphide (TIP) is the more stable phase compared to the other phases for the thallium phosphide compound.

In the present work, we report first principles calculations of the equilibrium lattice constant, bulk modulus and its pressure derivatives, the crystal density, the near-neighbor and nearest-neighbor distances. In addition, the threshold pressure of the phase transition, the volume collapses and the Debye temperature are also obtained. Our results are in general in good agreement with the previous results of the literature.

\section{Computational methods}

The first-principles calculations were performed by using pseudopotential plane-waves approach based on the density functional theory [4] and implemented in the ABINIT code [5-7]. ABINIT code is a package whose main program allows one to find the total energy, charge density, electronic structure, elastic, thermodynamic and optic properties of systems made of electrons and nuclei (molecules and periodic solids) within density functional theory, employing pseudopotentials and a plane-wave basis-set. It is a common project of the Universite Catholique de Louvain, Corning Incorporated, the Université de Liège and other contributors. 
The Hartwigsen Goedecker Hutter (HGH) relativistic separable dual space Gaussian pseudopotentials [8] in the context of the local density approximation are employed in the computation. The electron-electron interaction was studied within the local density approximation (LDA) by Teter-Pade parameterization [9], which reproduces the correlation scheme of Perdew and Wang [10].

A plane-wave basis set was used to solve the Kohn-Sham equations in the pseudopotential implementation of the DFTLDA. The Brillouin zone integrations were replaced by discrete summations over a special set of k-points, using the standard k-point technique of Monkhorst and Pack [11] where the k-point mesh used is $(8 \times 8 \times 8)$. The plane-wave energy cutoff to expand the wave functions was set to 90 Hartree. Careful convergence tests show that with these parameters relative energy converged to better than $10^{-5}$ Hartree.

\section{Results and discussions}

\subsection{Equilibrium lattice constant, bulk modulus and its pressure derivatives}

After obtaining the kinetic energy and the number of special k-points which gives the best convergence possible of the total energy; they were then used in the calculation of the total energy for various values of the lattice constant. The different values obtained of the total energy were then traced as a function of the unit cell volume. One can deduce the static structural properties such as the equilibrium lattice constant from the volume which gives the minimum energy, the bulk modulus, and its pressure derivatives using the following Murnaghan equation of state (EOS) [12].

$E(V)-E\left(V_{0}\right)=\frac{B_{0} V}{B_{0}^{\prime}}\left[\frac{\left(V_{0} / V\right)^{B_{0}^{\prime}}}{B_{0}^{\prime}-1}+1\right]-\frac{B_{0}^{\prime} V_{0}}{B_{0}^{\prime}-1}$

$B_{0}=V \frac{\partial^{2} E}{\partial V^{2}}=\frac{4}{9 a} \frac{\partial^{2} E}{\partial a^{2}}$

Where $B_{0}$ is the bulk modulus given by the relation (1-b) at zero pressure $(\mathrm{P}=0), V_{0}$ is the equilibrium volume, $E\left(V_{0}\right)$ is the energy corresponding to equilibrium volume, $B_{0}^{\prime}$ is the pressure derivative of the bulk modulus at $\mathrm{P}=0$ and, $a$ is the lattice constant. The figure 1 shows the evolution of the total energy as a function of the unit cell volume of both zincblende (B3) and Rock-salt (B1) phases of TlP compound. From the graphs connecting the total energy and relative volume one can clearly see that the (B3) phase is more stable than the (B1) phase.
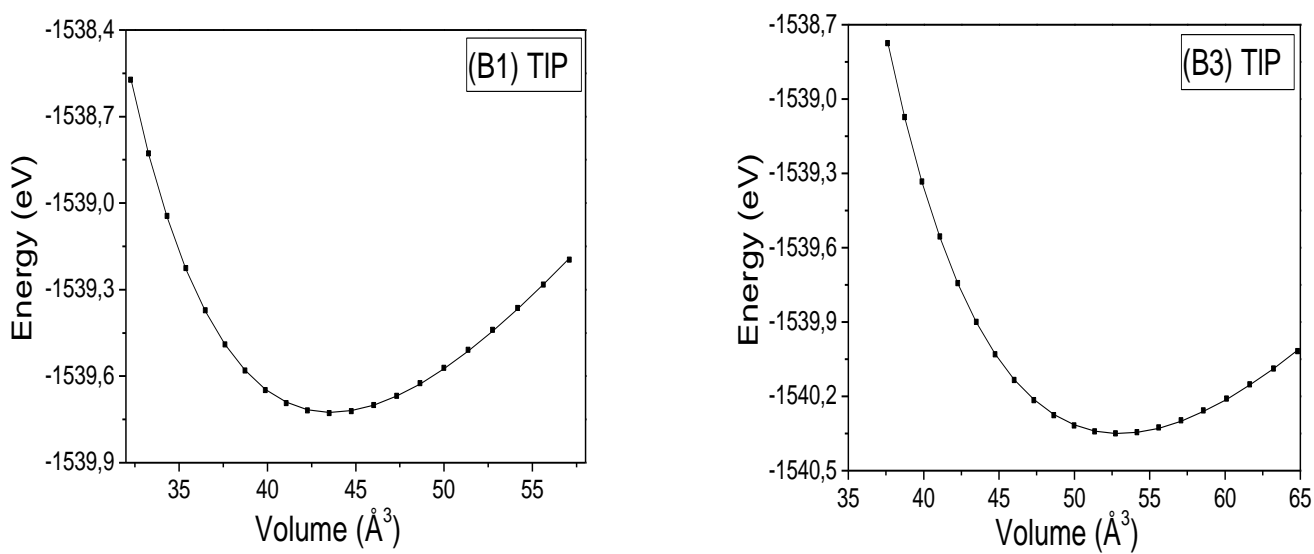

Fig. 1: Total Energy Variation with the Unit Cell Volume of both (B3) and (B1) Phases of TIP.

The values of the lattice parameter $\mathrm{a}_{0}$, bulk modulus $\mathrm{B}_{0}$, and its pressure derivative $\mathrm{B}_{0}{ }^{\prime}$ of both zincblende and Rock-salt phases are presented in table 1, and compared with the available theoretical data [2], [3], [13-17]. It is seen that, our calculated equilibrium lattice parameter $\mathrm{a}_{0}$ of zincblende phase is in excellent agreement with the previously calculated data $[2,3,13-17]$.

The value of lattice constant obtained by us, with the Murnaghan equation of state, deviates from the calculated [2], [13] LDA and [3] GGA values within $3.76 \%$ and $2.63 \%$ respectively. It can also be seen, that our calculated bulk modulus $\mathrm{B}_{0}$ obtained with the help of Eq. (1-b) $(58.076 \mathrm{GPa})$ is also in good agreement with the earlier calculations; its deviation is about $0.94 \%$ from the value calculated by Ciftci et al [2] LDA.

Our calculated equilibrium lattice parameter $\mathrm{a}_{0}$ of RS phase is also in concordance with the previously calculated data [2], [14]. The bulk modulus $\mathrm{B}_{0}$ of RS phase is also in good agreement with the other theoretical calculations; its deviation is about $6.77 \%$ from the value obtained by Ciftci et al [2] LDA. 
Table 1: Lattice Constants $\mathrm{a}_{0}$, Bulk Modulus $\mathrm{B}_{0}$ and its Pressure Derivative $\mathrm{B}_{0}{ }^{\prime}$ of TIP in Comparison With Other Theoretical Values [2], [3], [13-17].

\begin{tabular}{|c|c|c|c|c|c|}
\hline Phase & Parameter & Our work & & Other works & \\
\hline \multirow{4}{*}{ (B3) } & $\mathrm{a}_{0}(\AA)$ & 5.963 & $\begin{array}{l}5.747[2,13] \mathrm{LDA} \\
6.001[14] \mathrm{GGA}\end{array}$ & $\begin{array}{c}6.125[2] \mathrm{GGA} \\
5.96[15] \mathrm{LDA}\end{array}$ & $6.124[3,17] \mathrm{GGA}$ \\
\hline & \multirow{2}{*}{$\mathrm{B}_{0}(\mathrm{GPa})$} & \multirow[t]{2}{*}{50.076} & $57.536[2] \mathrm{LDA}$ & $45.975[2] \mathrm{GGA}$ & $46.75[3,17] \mathrm{GGA}$ \\
\hline & & & $71.3[13]$ & $53.3[14] \mathrm{GGA} \quad 57[15] \mathrm{LDA}$ & $80.46[16] \mathrm{LDA}$ \\
\hline & $\mathrm{B}_{0}^{\prime}$ & 4.425 & $\begin{array}{l}4.857[2] \mathrm{LDA} \\
4.5[14] \mathrm{GGA}\end{array}$ & $4.401[2] \mathrm{GGA}$ & 4.847[13]LDA \\
\hline \multirow{3}{*}{ (B1) } & $\mathrm{a}_{0}(\AA)$ & 5.588 & 5.574[2]LDA & 5.717[2]GGA & $5.599[14] \mathrm{GGA}$ \\
\hline & $\mathrm{B}_{0}(\mathrm{GPa})$ & 67.223 & 72.102[2]LDA & 53.542[2]GGA & $62.4[14] \mathrm{GGA}$ \\
\hline & $\mathrm{B}_{0}^{\prime}$ & 4.779 & 4.811[2]LDA & 4.887[2]GGA & 4.6[14]GGA \\
\hline
\end{tabular}

\subsection{Pressure phase transition and pressure-volume curve}

Under the application of hydrostatic pressure, the more stable solid phase is destabilized, and consequently, a structural phase transition occurs, which is usually accompanied by a structural change in crystallographic data of the crystalline solid, such as lattice parameters and atomic occupied positions. For thallium phosphide compound, the phase transition is from the zincblende (B3) to Rock-salt (B1) [3], [17]. To estimate the numerical value of the threshold pressure of the phase transition, for $\mathrm{A}^{\mathrm{III}} \mathrm{B}^{\mathrm{V}}$ group semiconductors, we can use the following emperical formula [18]:

$P_{T}=76.2-11.5 a$

Where: $P_{T}$ is the threshold pressure of the phase transition (in GPa) and a is the equilibrium lattice parameter (in $\AA$ ).

The numerical value of the threshold pressure of the phase transition for (B3) TIP compound is given in table 2 and compared with the available theoretical data [2, 14, and 16].

Table 2: The Threshold Pressure of the Phase Transition for (B3) TIP Compound, in Comparison with Other Theoretical Values [2], [14], and [16].

\begin{tabular}{ccccc}
\hline Parameter & Our work & \multicolumn{3}{c}{ Other works } \\
\hline$P_{T}(\mathrm{GPA})$ & 7.626 & $4.47[2]$ & $7.29[14] \mathrm{GGA}$ & $7.2[16]$
\end{tabular}

It can be seen, that our calculated values of $P_{T}$ obtained, are in general in good agreement with the earlier calculations; the deviation from the calculated value of Shi et al. [14] is only about $4.6 \%$.

In order to further validate the reliability and accuracy of our calculated properties, the calculated unit cell volumes under a series of applied hydrostatic pressures were used to construct the P-V data set, which was subsequently fitted by the Murnaghan equation [19], written below:

$P(V)=\frac{3}{2} B_{0}\left[\left(\left(\frac{V_{0}}{V}\right)^{\frac{7}{3}}-\left(\frac{V_{0}}{V}\right)^{\frac{5}{3}}\right)\right]\left\{1+\frac{3}{4}\left(B_{0}{ }^{\prime}-4\right)\left[\left(\left(\frac{V_{0}}{V}\right)^{\frac{2}{3}}-1\right)\right]\right\}$

Where: $B_{0}$ is the bulk modulus at $P=0, B_{0}^{\prime}$ is its pressure derivative at $P=0, \mathrm{~V}_{0}$ is the volume of unit cell, is fixed at the value determined from the $P=0$ data, and $\mathrm{V}$ is the volume of unit cell at $P \neq 0$.

In order to show how the structural parameters behave under hydrostatic pressure, the equilibrium geometries of (B3) TIP unit cells were computed at fixed values of applied hydrostatic pressure in the 0 to $7 \mathrm{GPa}$ range, where at each pressure value, a complete optimization for the volume unit cells was performed. In figure 2, we plot the variation of pressure versus unit cell volume, and versus the relative unit cell volume of (B3) TIP compound.
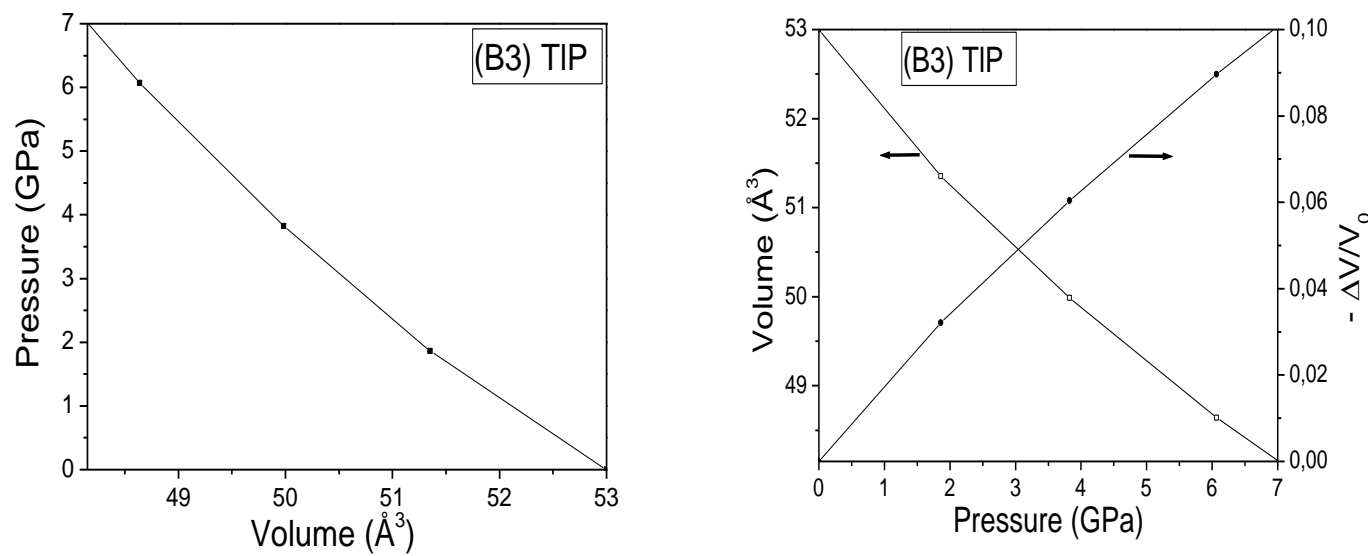

Fig. 2: Pressure-Volume Dependence and the Relative Unit Cell Volume versus Applied Pressure of (B3) TIP. 
As shown in figure 2, the volume decreases gradually with the increasing of the hydrostatic pressure, where the volume takes the value about $53 \AA^{3}$ at zero-pressure and reaches the value $48.15 \AA^{3}$ at $\mathrm{P}=7 \mathrm{GPa}$.

The equation of state curves of thallium phosphide compound (plotted between $\mathrm{V}(\mathrm{P}) / \mathrm{V}(0)$ and pressure) for both the B3 and B1 structures obtained in this work is in fairly good agreement with the other theoretical results [14]. The calculated values of the volume collapses $[\Delta \mathrm{V}(\mathrm{P}) / \mathrm{V}(0)]$ are also close to their observed data.

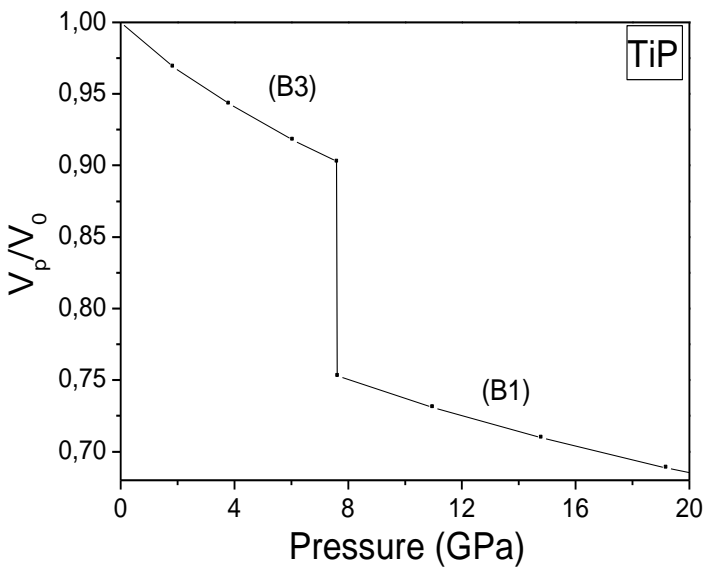

Fig. 3: Variations of the Ratio $\mathrm{V}_{\mathrm{p}} / \mathrm{V}_{0}$ of (B3) and (B1) TIP with Hydrostatic Pressure.

From these curves, we can estimate the volume collapse in the point of transition. The results of numerical value of the volume collapse for (B3) TIP compound is given in table 3 and compared with the available theoretical data [14], [16].

Table 3: Volume Collapse for (B3) TIP Compound, In Comparison with the Available Theoretical Values [14, 16].

\begin{tabular}{cccc}
\hline Parameter & Our work & \multicolumn{2}{c}{ Other works } \\
\hline Volume collapse (\%) & 16.59 & $17.45[14] \mathrm{GGA}$ & $15.78[16]$
\end{tabular}

It is seen that, our calculated value of the volume collapse is in excellent agreement with the previously calculated data [14], [16]. The value obtained by us, deviates from the calculated [14] GGA and [16] values within $4.93 \%$ and 5.13\% respectively.

\subsection{Bond length and crystal density}

In a conventional unit cell of the cubic-zinc blende lattice there are four molecules, and if a lattice constant is available, the calculation of the crystal density $\mathrm{g}$ is easy, this later is given by the following formula [20].

$$
g=\frac{Z \cdot M \cdot u}{a \cdot b \cdot c \sqrt{\sin ^{2} \alpha+\sin ^{2} \beta+\sin ^{2} \delta-2(1-\cos \alpha \cdot \cos \beta \cdot \cos \delta)}}
$$

Where $\mathrm{Z}$ is the number of formula units in a crystal unit cell, $\mathrm{M}$ is the molecular weight of a formula unit in amu, $\mathrm{u}$ is weight of an amu, a , b and $\mathrm{c}$ are unit cell axes lengths and $\alpha, \beta$ and $\delta$ are unit cell axes angles.

The crystal density of (B3) TIP compound at zero-pressure is: $\mathrm{g}=7.373 \mathrm{~g} / \mathrm{cm}^{3}$. The calculated crystal density versus the hydrostatic pressure of the (B3) TIP is plotted in figure 4.

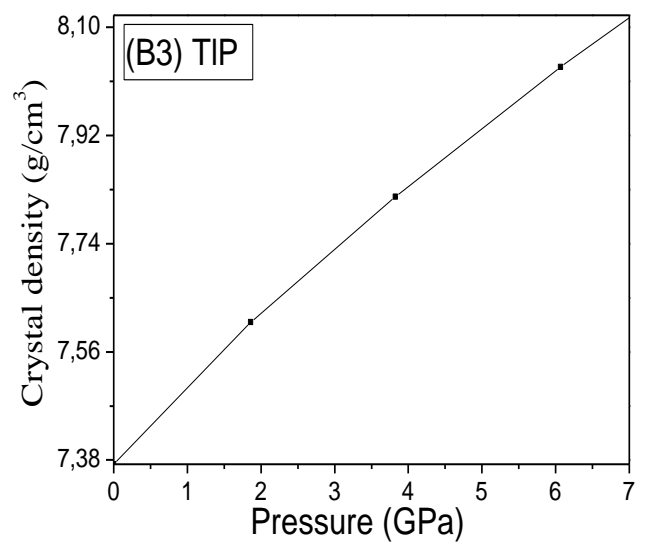

Fig. 4: Crystal Density versus the Hydrostatic Pressure of (B3) TIP Compound. 
Nearest-neighbors (NN) named also bond lengths d, it have been defined as the average distance between nearestneighbour atoms. For example, in our case (in the TIP compound), the bond length is defined as the average distance between $\mathrm{Ti}$ (group III element) and $\mathrm{P}$ (group $\mathrm{V}$ element) atoms.

The spacing between nearest-neighbors atoms (bond length) is equal to $\sqrt{3} \mathrm{a}_{0} / 4$, and the spacing between the nearneighbor atoms is equal to $\mathrm{a}_{0} / \sqrt{2}$ [21]. For (B3) TIP compound, the calculated nearest-neighbor and near-neighbor distances are $2.582 \AA$ and $4.216 \AA$ respectively.

The crystal density of (B1) TIP compound at zero-pressure is also obtained, it's equal to: $8.959 \mathrm{~g} / \mathrm{cm}^{3}$. In the case of the Rock salt phase, there is only one type of first-neighbor cation-anion bond distance (with six bonds for each atom), and the spacing of the bond length is equal to $\mathrm{a}_{0} / 2$, so it's equal to: $2.794 \AA$.

\section{4. Debye temperature}

Adachi [18] proposed a linear relation between the Debye temperature $\left(\theta_{\mathrm{D}}\right)$ and the equilibrium lattice parameter $\left(\mathrm{a}_{0}\right)$ for $\mathrm{A}^{\mathrm{III}-} \mathrm{B}^{\mathrm{V}}$ semiconductors; it is given by the following formula:

$\ln \Theta_{\mathrm{D}}=10.53-0.834 \mathrm{a}_{0}$

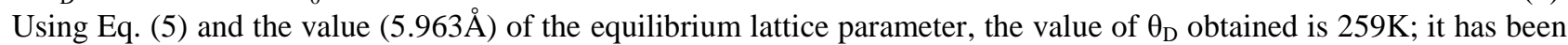
listed in table 4 and compared with the available theoretical data [2, 14, and 16].

Table 4: Debye Temperature of (B3) TIP Compound, In Comparison with the Available Theoretical Values $[2,14,16]$.

\begin{tabular}{ccccc}
\hline Parameter & Our work & \multicolumn{4}{c}{ Other works } \\
\hline$\theta_{\mathrm{D}}(\mathrm{K})$ & 259 & $302[2]$ & $256.4[14]$ & $346[16]$ \\
\hline
\end{tabular}

It can also be seen, that our calculated value of the Debye temperature is also in good agreement with the earlier calculations; its deviation is about $1 \%$ from the value calculated by Shi et al [14].

\section{Conclusion}

A summary of the remarks and results obtained in this work are given below:

The calculated equilibrium lattice constant, the bulk modulus and its pressure derivative at zero-pressure of both zinc blende (B3) and Rock-salt (B1) phases of TIP compound are in very good agreement with the previous results reported in the literature. The bond length and crystal density are also obtained.

The phase transition from the zinc blende (B3) to Rock-salt (B1) is obtained; it's in excellent agreement with the previous results of the literature. The effect of hydrostatic pressure on crystal density of B3 phase is obtained. The calculated values of the volume collapses and the Debye temperature are in fairly good agreement with the other theoretical results.

\section{References}

[1] R. R. Reddy, Y. Nazeer Ahammed, P. Abdul Azeem, K. Rama Gopal, B. Sasikala Devi \&T. V. R. Rao, "Dependence of Physical Parameters of Compound Semiconductors on Refractive Index", Defense Science Journal, Vol. 53, No. 3, (2003), pp. $239-248$. http://www.publications.drdo.gov.in/ojs/index.php/dsj/article/view/2272.

[2] Y. O. Ciftci, K. Colakoglu \& E. Deligoz, "A First Principles Studies on TIX (X=P, As)", Central European Journal of Physics, Vol. 6, No. 4, (2008), pp. 802-807. http://link.springer.com/article/10.2478\%2Fs11534-008-0109-y. http://dx.doi.org/10.2478/s11534-008-0109-y

[3] N. Saidi-Houat, A. Zaoui \& M. Ferhat, "Structural stability of thallium-V compounds", Journal of Physics: Condensed Matter, Vol. 19, No. 10, (2007), pp. 106221 (18pp). http://iopscience.iop.org/0953-8984/19/10/106221/.

[4] E. Engel \& R. M Dreizler, "Density Functional Theory", Springer-Verlag, New York, (2011). http://dx.doi.org/10.1007/978-3-642-14090-7.

[5] X Gonze, J.-M. Beuken a, R. Caracas, et al, "First-principles computation of material properties: the ABINIT software project" Computational Materials Science, Vol. 25, (2002), pp. 478-492. www.researchgate.net/computationmaterial/9fcfd50c5f4ec3f833.pdf.

[6] X. Gonze, G. M. Rignanese, M. Verstraete, et al. "A brief introduction to the ABINIT software package", Zeitschrift für Kristallographie, Vol.220, No.5-6, (2005), pp. 558-562. dx.doi.org/10.1524/zkri.220.5.558.65066.

[7] The ABINIT code is a common project of the Universite Catholique de Louvain, Corning Incorporated, and other contributors (URL http://www.abinit.org).

[8] S. Goedecker \& J. Hutter, "Relativistic separable dual-space Gaussian pseudopotentials from H to Rn", Physical Review B, Vol. 58, (1998), pp. 3641-3662. http://dx.doi.org/10.1103/PhysRevB.58.3641. http://dx.doi.org/10.1103/PhysRevB.58.3641.

[9] S. Goedecker, M. Teter, \& J. Hutter, "Separable Dual-Space Gaussian Pseudopotentials", Physical Review B, Vol. 54, No. 3, (2003), pp. 1703-1710. https://trac.cc.jyu.fi/projects/toolbox/raw.../wiki/../GTH_96.pdf. http://dx.doi.org/10.1103/PhysRevB.54.1703.

[10] J. P. Perdew \& Y. Wang, "Accurate and simple analytic representation of the electron-gas correlation energy", Physical Review B, Vol. 45, No. 32, (2003), pp. 13244-13249. link.aps.org/doi/10.1103/PhysRevB.45.13244.

[11] H .J. Monkhorst \& J. D. Pack, "Special points for Brillouin-zone integrations", Physical Review B, Vol.13, No.12, (1976), pp. 5188-5192. DOI: http://dx.doi.org/10.1103/PhysRevB.13.5188.

[12] F. Murnaghan, "The Compressibility of Media under Extreme Pressures", Proceedings of the National Academy of Sciences of the United States of America, Vol. 30, No. 9, (1944), pp. 244-247. doi:10.1073/pnas.30.9.244. http://dx.doi.org/10.1073/pnas.30.9.244.

[13] S. Q. Wang \& H. Q. Yet, "Plane-wave pseudopotential study on mechanical and electronic properties for IVand III-V crystalline phases with zinc-blende structure", Physical Review B, Vol.66, (2002), pp. 235111 (7pp). link.aps.org/doi/10.1103/PhysRevB.66.235111. 
[14] L. Shi, Y. Duan \& L. Qin, "Structural phase transition, electronic and elastic properties in TIX (X = N, P, as) compounds: Pressure-induced effects", Computational Materials Science, Vol.50, (2010), pp. 203-210. www.sciencedirect.com/science/article/pii/S0927025610004532. http://dx.doi.org/10.1016/j.commatsci.2010.07.027.

[15] M. van Schilfgaarde, An-Ban Chen, S. Krishnamurthy \& Arden Sher, "InT1P - a proposed infrared detector material", Applied Physics Letters, Vol.65, No.21, (1994), pp. 2714-2716. http://dx.doi.org/10.1063/1.112567. http://dx.doi.org/10.1063/1.112567.

[16] S. Singh \& M. Sarwan, "High pressure phase transition and elastic behavior of TIX (X=N, P, as) semiconductors", Journal of Physics and Chemistry of Solids, Vol.74, (2013), pp. 487-495. www.sciencedirect.com/science/article/pii/S0022369712003617. http://dx.doi.org/10.1016/j.jpcs.2012.11.016

[17] M. Ferhat \& A. Zaoui, "Do all III-V compounds have the zinc-blende or wurtzite ground state structure?" Applied Physics Letters, Vol.88, No.16, (2006), pp. 161902-161904. http://dx.doi.org/10.1063/1.2196050.

[18] S. Adachi, "Properties of Group-IV, III-V and II-VI Semiconductors", John Wiley \& Sons, England, (2005). http://dx.doi.org/10.1002/0470090340.

[19] F. Birch, "Finite strain isotherm and velocities for single-crystal and polycrystalline $\mathrm{NaCl}$ at high pressures and $300^{\circ} \mathrm{K}$ ", Journal of Geophysical Research, Vol. 83, No. B3, (1978), pp. 1257 - $1268 . \quad$ DOI: $10.1029 / \mathrm{JB} 083 \mathrm{iB} 03$ p01257 http://dx.doi.org/10.1029/JB083iB03p01257.

[20] W. J. Tropf, M. F. Thomas \& T. J. Harris, "Properties of crystals and glasses", Handbook of Optics, Vol. IV, McGraw-Hill, New York, (2004).

[21] S. Adachi, "Physical properties of III-V semiconductor compounds", New York: John Wiley \& Sons, (1992). http://dx.doi.org/10.1002/352760281X. 